Bond University

Research Repository

\title{
Urinary alkalisation for uncomplicated urinary tract infection (Protocol)
}

O'Kane, Dermot B; Dave, Sameer; Gore, Neel; Patel, Farhaan; Hoffmann, Tammy C; Del Mar, Chris B

Published in:

Cochrane Database of Systematic Reviews

DOI:

10.1002/14651858.CD010745

Licence:

Other

Link to output in Bond University research repository.

Recommended citation(APA):

O'Kane, D. B., Dave, S., Gore, N., Patel, F., Hoffmann, T. C., \& Del Mar, C. B. (2013). Urinary alkalisation for uncomplicated urinary tract infection (Protocol). Cochrane Database of Systematic Reviews, 2013(10), 1-12. [CD010745]. https://doi.org/10.1002/14651858.CD010745

\section{General rights}

Copyright and moral rights for the publications made accessible in the public portal are retained by the authors and/or other copyright owners and it is a condition of accessing publications that users recognise and abide by the legal requirements associated with these rights.

For more information, or if you believe that this document breaches copyright, please contact the Bond University research repository coordinator. 


\section{(E) Cochrane Library}

Cochrane Database of Systematic Reviews

\section{Urinary alkalisation for uncomplicated urinary tract infection (Protocol)}

O’Kane DB, Dave SK, Gore N, Patel F, Hoffmann T, Del Mar CB

O'Kane DB, Dave SK, Gore N, Patel F, Hoffmann T, Del Mar CB.

Urinary alkalisation for uncomplicated urinary tract infection.

Cochrane Database of Systematic Reviews 2013, Issue 10. Art. No.: CD010745.

DOI: 10.1002/14651858.CD010745.

www.cochranelibrary.com 
TABLE OF CONTENTS

HEADER . . . . . . . . . . . . . . . . . . . . . . . . . . . . . . . . . . . . 1

ABSTRACT . . . . . . . . . . . . . . . . . . . . . . . . . . . . . . . . . . . . . . 1

BACKGROUND . . . . . . . . . . . . . . . . . . . . . . . . . . . . . . . . . . . .

OBJECTIVES . . . . . . . . . . . . . . . . . . . . . . . . . . . . . . . . . . . . . . . . . .

METHODS . . . . . . . . . . . . . . . . . . . . . . . . . . . . . . . . . . . . . . .

ACKNOWLEDGEMENTS . . . . . . . . . . . . . . . . . . . . . . . . . . . . . . . . . . . . . . . .

REFERENCES . . . . . . . . . . . . . . . . . . . . . . . . . . . . . . . . . . . . . . . 5

APPENDICES . . . . . . . . . . . . . . . . . . . . . . . . . . . . . . . . . . . . . 6

CONTRIBUTIONS OF AUTHORS . . . . . . . . . . . . . . . . . . . . . . . . . . . . . . . . . . . 10

DECLARATIONS OF INTEREST . . . . . . . . . . . . . . . . . . . . . . . . . . . . . . . . . 10 


\title{
[Intervention Protocol]
}

\section{Urinary alkalisation for uncomplicated urinary tract infection}

\author{
Dermot B O’Kane ${ }^{1}$, Sameer K Dave ${ }^{1}$, Neel Gore ${ }^{1}$, Farhaan Patel ${ }^{1}$, Tammy Hoffmann ${ }^{1}$, Chris B Del Mar ${ }^{1}$ \\ ${ }^{1}$ Centre for Research in Evidence-Based Practice (CREBP), Bond University, Gold Coast, Australia \\ Contact address: Chris B Del Mar, Centre for Research in Evidence-Based Practice (CREBP), Bond University, Gold Coast, QLD, \\ Australia.cdelmar@bond.edu.au.
}

Editorial group: Cochrane Kidney and Transplant Group.

Publication status and date: New, published in Issue 10, 2013.

Citation: O'Kane DB, Dave SK, Gore N, Patel F, Hoffmann T, Del Mar CB. Urinary alkalisation for uncomplicated urinary tract infection. Cochrane Database of Systematic Reviews 2013, Issue 10. Art. No.: CD010745. DOI: 10.1002/14651858.CD010745.

Copyright (C) 2013 The Cochrane Collaboration. Published by John Wiley \& Sons, Ltd.

\begin{abstract}
A B S T R A C T
This is the protocol for a review and there is no abstract. The objectives are as follows:

We aim to look at the benefits and harms of the use of urinary alkalisers for the treatment of uncomplicated UTIs in adult women.
\end{abstract}

\section{B A C K G R O U N D}

\section{Description of the condition}

Urinary tract infection (UTI) is the most common form of bacterial infection in women, most of which are uncomplicated (Foxman 2003). UTIs are considered uncomplicated in the absence of urinary tract abnormalities, obstruction or resistant pathogens, pregnancy, immunocompromised state, or acute pyelonephritis. The term 'uncomplicated' is not applied to UTIs in adult men.

Standard conventional management of suspected UTI is to confirm presence of either bacteria or white cells in the urine, and treat with antibiotics. However, studies have challenged this approach. Whatever the role of antibiotics, women often seek relief from the symptoms of UTI until either the infection is cleared with antibiotics or resolves spontaneously. Uncomplicated UTI often spontaneously remit without antibiotics: a recent pilot randomised controlled trial (RCT) found that symptomatic treatment for uncomplicated UTI was non-inferior to antimicrobial therapy (Bleidorn 2010). Nonetheless, an earlier study also found that women with culture-negative symptoms of UTI respond to antibiotics (Richards 2005).

\section{Description of the intervention}

Several clinical guidelines for UTI treatment advise first-line use of antibiotics (ACOG 2008; Grabe 2010; IDSA/ESMI 2011). However, until infection has cleared (due to either antimicrobial therapy or spontaneous remit), symptoms may be troublesome. The use of urinary alkalisers for the symptomatic treatment of uncomplicated UTI is very common in some countries. For example, more than one million units of urinary alkalisers are sold in Australia annually; many of these are used specifically for UTIs and acute culture-negative cystitis. Use of urinary alkalisers for the symptomatic treatment of UTI and cystitis appear in MIMS Australia and other national formularies (eMC 2013; MIMS 2013). Use is also widely promoted by primary healthcare practitioners. Literature supporting benefits from use of these agents is sparse; some guidelines specifically state that they are not recommended (NICE 2009). 


\section{How the intervention might work}

Urinary alkalisers primarily work to raise urine $\mathrm{pH}$, which in theory, aids in the symptomatic treatment of dysuria. Dysuria and urinary frequency are the most common and bothersome symptoms of UTI and acute culture-negative cystitis (Munday 1990; Spooner 1984).

Urinary pathogens, such as Proteus mirabilis can also increase urinary $\mathrm{pH}$ and are associated with symptoms of dysuria and urinary frequency (Franz 1999); however, it has been suggested elsewhere that raising urine $\mathrm{pH}$ does not affect the sensation of dysuria (Brumfitt 1990).

\section{Why it is important to do this review}

Uncomplicated UTI is very common, and imposes significant financial burden. In the US, UTI is responsible for over seven million physician visits annually, and account for the use of approximately $15 \%$ of all community-prescribed antibiotics. The total annual estimated cost of antibiotics for UTI in the USA exceeds one billion USD (Foxman 2002; Mazzulli 2002). The advent of increasing numbers of drug resistant organisms means that avoidance of unnecessary antibiotics is important.

Urinary alkalisers are widely used globally for symptomatic relief of UTI and acute culture-negative cystitis. There is however a paucity of good evidence to support their use. There is also conflicting evidence surrounding urinary alkalinisation for antimicrobial eradication in UTI. Some studies show benefit of concomitant urinary alkalisers and antibiotic use, with reduced antibiotic MIC when urine is at alkaline $\mathrm{pH}$. Other studies show that urine $\mathrm{pH}$ within the acidic range is favourable, as it can have a bactericidal effect (Burian 2012; Carlsson 2003; Zhanel 1991). Although we aim to focus on symptom relief in UTI, antimicrobial eradication may also be important, as prolonged positive urine culture may lead to a prolongation of symptoms. If we find insufficient evidence surrounding these agents, then this sets a research agenda.

\section{O B J E C T I VES}

We aim to look at the benefits and harms of the use of urinary alkalisers for the treatment of uncomplicated UTIs in adult women.

\section{METHODS}

\section{Criteria for considering studies for this review}

\author{
Types of studies
}

All RCTs and quasi-RCTs (RCTs in which allocation to treatment was obtained by alternation, use of alternate medical records, date of birth or other predictable methods) looking at the use of urinary alkalisers (of any type) for the symptomatic relief of UTI.

\section{Types of participants}

\section{Inclusion criteria}

We will include women aged 16 years or over with symptoms of uncomplicated UTI or cystitis including urinary frequency and dysuria. Participants will be included regardless of whether diagnoses were made from positive urine dipstick test results or positive urine culture. Women with recurrent UTI may be included if they were not symptomatic in the previous two weeks.

\section{Exclusion criteria}

- Complicated UTIs, such as those requiring hospital admission, infections associated with fevers or rigours, multidrug resistant pathogens Neisseria gonorrhoeae and Chlamydia trachomatis urethritis, urinary tract abnormalities, urinary tract calculi or urinary tract obstruction

- Immunocompromising conditions

- Acute pyelonephritis

- UTI symptoms in the previous two weeks

- Chronic conditions such as interstitial cystitis, painful bladder syndrome, chronic pelvic pain syndrome.

\section{Types of interventions}

Any urinary alkaliser used exclusively or non-exclusively for the treatment of symptoms of UTI will be included. We plan to compare:

- Urinary alkalisers versus placebo/no treatment

- Urinary alkalisers versus antibiotics

- Urinary alkalisers + antibiotics versus antibiotics alone

- Urinary alkalisers versus anti-inflammatories.

\section{Types of outcome measures}

\section{Primary outcomes}

- Early and late symptoms (at days 1 to 4 and days 5 to 10 ): dysuria, urinary frequency, and abdominal pain

- Any adverse events: worsening of UTI, progression to complicated UTI, need for hospitalisation or intravenous antibiotics. 


\section{Secondary outcomes}

- Duration of symptoms

- Severity of symptoms (negligible, mild, moderate, severe) as measured on days 1 to 4 and days 5 to 10

- Number of return visits to the GP

- Days absent from work

- Bacterial eradication.

\section{Search methods for identification of studies}

\section{Electronic searches}

We will search the Cochrane Renal Group's Specialised Register through contact with the Trials' Search Co-ordinator using search terms relevant to this review. The Cochrane Renal Group's Specialised Register contains studies identified from the following sources.

1. Monthly searches of the Cochrane Central Register of Controlled Trials (CENTRAL)

2. Weekly searches of MEDLINE OVID SP

3. Handsearching of renal-related journals and the proceedings of major renal conferences

4. Searching of the current year of EMBASE OVID SP

5. Weekly current awareness alerts for selected renal journals

6. Searches of the International Clinical Trials Register (ICTRP) Search Portal and ClinicalTrials.gov.

Studies contained in the Specialised Register are identified through search strategies for CENTRAL, MEDLINE, and EMBASE based on the scope of the Cochrane Renal Group. Details of these strategies, as well as a list of handsearched journals, conference proceedings and current awareness alerts, are available in the Specialised Register section of information about the Cochrane Renal Group. See Appendix 1 for search terms used in strategies for this review.

\section{Searching other resources}

1. Reference lists of clinical practice guidelines, review articles and relevant studies.

2. Letters seeking information about unpublished or incomplete studies to investigators known to be involved in previous studies.

\section{Data collection and analysis}

\section{Selection of studies}

The search strategy described will be used to obtain titles and abstracts of studies that may be relevant to the review. The titles and abstracts will be screened independently by two authors, who will discard studies that are not applicable; however studies and reviews that might include relevant data or information on studies will be retained initially. Two authors will independently assess retrieved abstracts and, if necessary the full text, of these studies to determine which studies satisfy the inclusion criteria.

\section{Data extraction and management}

Data extraction will be carried out independently by two authors using standard data extraction forms. Studies reported in nonEnglish language journals will be translated before assessment. Where more than one publication of one study exists, reports will be grouped together and the publication with the most complete data will be used in the analyses. Where relevant outcomes are only published in earlier versions these data will be used. Any discrepancy between published versions will be highlighted.

\section{Assessment of risk of bias in included studies}

The following items will be independently assessed by two authors using the risk of bias assessment tool (Higgins 2011) (see Appendix 2).

- Was there adequate sequence generation (selection bias)?

- Was allocation adequately concealed (selection bias)?

- Was knowledge of the allocated interventions adequately prevented during the study (detection bias)?

- Participants and personnel

- Outcome assessors

- Were incomplete outcome data adequately addressed (attrition bias)?

- Are reports of the study free of suggestion of selective outcome reporting (reporting bias)?

- Was the study apparently free of other problems that could put it at a risk of bias?

\section{Measures of treatment effect}

For dichotomous outcomes such as resolution of symptoms by day three and day seven (dysuria, urinary frequency, abdominal pain) and progression to complicated UTI, results will be expressed as risk ratio (RR) with $95 \%$ confidence intervals (CI). Where continuous scales of measurement are used to assess the effects of treatment, such as duration of symptoms, severity of symptoms (negligible, mild, moderate, severe; 0 to 3), the mean difference (MD) will be used, or the standardised mean difference (SMD) if different scales have been used.

\section{Unit of analysis issues}

In relation to cluster-RCTs, only studies where analyses were made at the same level as allocation (and using summary measurements for each cluster) will be included. Cluster-RCTs may also be included if statistical methods were employed to deal with analysis 
at the individual level, which can account for the clustering of the data, and that these statistical methods were clearly outlined in the methods, and are sound.

Data from cross-over RCTs may be assessed, but only the first randomisation period will be included. Complete cross-over data are not appropriate for the intervention under review.

Urinary alkalisers could be investigated alone or in combination with another agent(s) where the only difference between groups was addition of a urinary alkaliser. Where possible we aim to combine groups with the same intervention and create a single pairwise comparison.

\section{Dealing with missing data}

Any further information required from original authors will be requested by written correspondence (e.g. emailing or writing to corresponding author) and any relevant information obtained in this manner will be included in the review. Evaluation of important numerical data such as screened, randomised patients as well as intention-to-treat, as-treated and per-protocol population will be carefully performed. Attrition rates, for example drop-outs, losses to follow-up and withdrawals will be investigated. Issues of missing data and imputation methods (e.g. last-observation-carriedforward) will be critically appraised (Higgins 2011).

\section{Assessment of heterogeneity}

Heterogeneity will be analysed using a $\mathrm{Chi}^{2}$ test on $\mathrm{N}-1$ degrees of freedom, with an alpha of 0.05 used for statistical significance and with the $\mathrm{I}^{2}$ test (Higgins 2003). $\mathrm{I}^{2}$ values of $25 \%, 50 \%$ and $75 \%$ correspond to low, medium and high levels of heterogeneity.

\section{Assessment of reporting biases}

If possible, funnel plots will be used to assess for the potential existence of small study bias (Higgins 2011).

\section{Data synthesis}

Data will be pooled using the random-effects model but the fixedeffect model will also be used to ensure robustness of the model chosen and susceptibility to outliers.

\section{Subgroup analysis and investigation of heterogeneity}

Subgroup analysis will be used to explore possible sources of heterogeneity (e.g. participants, interventions and study quality). Heterogeneity among participants could be related to age, urinary pathogen (including urinary bacterial cell counts), chronicity of symptoms before seeking medical advice, and history of urinary symptoms (including recurrent UTIs). If data are available we will perform a subgroup analysis comparing the use of urinary alkalisers in sporadic and recurrent UTIs. Heterogeneity in treatments could be related to prior or concomitant (or both) agent(s) used and the agent, dose and duration of therapy. These could include antibiotics, analgesics and anti-inflammatory medications. We plan to perform a subgroup analysis comparing doses and duration of urinary alkaliser therapy. Adverse effects will be tabulated and assessed using descriptive techniques, because they are likely to be different for the various agents used. Where possible, the risk difference with $95 \%$ CI will be calculated for each adverse effect, either compared with no treatment or another agent.

\section{Sensitivity analysis}

We will perform sensitivity analyses in order to explore the influence of the following factors on effect size.

- Repeating the analysis excluding unpublished studies

- Repeating the analysis taking account of risk of bias, as specified

- Repeating the analysis excluding any very long or large studies to establish how much they dominate the results

- Repeating the analysis excluding studies using the following filters: diagnostic criteria, language of publication, source of funding (industry versus other), and country.

\section{ACKNOWLEDGEMENTS}

We would like to thank the referees for their assistance, comments and feedback. 


\section{REFERE N C E S}

\section{Additional references}

ACOG 2008

American College of Obstetricians and Gynecologists. ACOG Practice Bulletin No. 91: Treatment of urinary tract infections in nonpregnant women. Obstetrics \& Gynecology 2008;111(3):785-94. [MEDLINE: 18310389]

Bleidorn 2010

Bleidorn J, Gagyor I, Kochen M, Wegscheider K, HummersPradier E. Symptomatic treatment (ibuprofen) or antibiotics (ciprofloxacin) for uncomplicated urinary tract infection?-results of a randomized controlled pilot trial. BMC Medicine 2010;8:30. [MEDLINE: 20504298]

\section{Brumfitt 1990}

Brumfitt W, Hamilton-Miller JM, Cooper J, Raeburn A. Relationship of urinary $\mathrm{pH}$ to symptoms of 'cystitis'. Postgraduate Medical Journal 1990;66(779):727-9. [MEDLINE: 2235803]

Burian 2012

Burian A, Erdogan Z, Jandrisitis C, Zeitlinger M. Impact of $\mathrm{pH}$ on activity of trimethoprim, fosfomycin, amikacin colistin and ertapenem in human urine. Pharmacology 2012;90(5-6):281-7. [MEDLINE: 23037005]

Carlsson 2003

Carlsson S, Govoni M, Wiklund NP, Weitzberg E, Lundberg JO. In vitro evaluation of a new treatment for urinary tract infections caused by nitrate-reducing bacteria. Antimicrobial Agents \& Chemotherapy 2003;47(12):3713-8. [MEDLINE: 14638471]

eMC 2013

electronic Medicines Compendium (eMC). http:// www.medicines.org.uk/emc (accessed 4 September 2013).

Foxman 2002

Foxman B. Epidemiology of urinary tract infections: incidence, morbidity, and economic costs. American Journal of Medicine 2002;113 Suppl 1A:5S-13S. [MEDLINE: 12113866]

\section{Foxman 2003}

Foxman B, Brown P. Epidemiology of urinary tract infections transmission and risk factors, incidence, and costs. Infectious Disease Clinics of North America 2003;17 (2):227-41. [MEDLINE: 12848468]

\section{Franz 1999}

Franz M, Hörl WH. Common errors in diagnosis and management of urinary tract infection. I: Pathophysiology and diagnostic techniques. Nephrology Dialysis Transplantation 1999;14(11):2746-53. [MEDLINE: 10534527]

Grabe 2010

Grabe M, Bjerklund-Johansen TE, Botto H, Wullt B, Çek M, Naber KG, et al. Guidelines on urological infections: Uncomplicated UTIs in adults. European Association of Urology, 2010. http://www.uroweb.org/
gls/pdf/Urological\%20Infections\%202010.pdf (accessed 4 September 2013).

\section{Higgins 2003}

Higgins JP, Thompson SG, Deeks JJ, Altman DG.

Measuring inconsistency in meta-analyses. BMJ 2003;327

(7414):557-60. [MEDLINE: 12958120]

\section{Higgins 2011}

Higgins JP, Green S (editors). Cochrane Handbook for Systematic Reviews of Interventions Version 5.1.0 [updated March 2011]. The Cochrane Collaboration, 2011.

Available from www.cochrane-handbook.org.

\section{IDSA/ESMI 2011}

Gupta K, Hooton TM, Naber KG, Wullt B, Colgan R, Miller LG, et al. International clinical practice guidelines for the treatment of acute uncomplicated cystitis and pyelonephritis in women: A 2010 update by the Infectious Diseases Society of America and the European Society for Microbiology and Infectious Diseases. Clinical Infectious Diseases 2011;52(5):e103-20. [MEDLINE: 21292654]

\section{Mazzulli 2002}

Mazzulli T. Resistance trends in urinary tract pathogens and impact on management. Journal of Urology 2002;168(4 Pt 2):1720-2. [MEDLINE: 12352343]

MIMS 2013

Uracol, Citralite, Citravescent sachets, Uricosal. MIMS Australia, 2013. Available from http://www.mims.com.au/.

Munday 1990

Munday PE, Savage S. Cymalon in the management of urinary tract symptoms. Genitourinary Medicine 1990;66 (6):461. [MEDLINE: 2265846]

\section{NICE 2009}

National Institute of Health and Care Excellence. Urinary tract infection (lower) - women. NICE Guidelines, 2009. http://cks.nice.org.uk/urinary-tract-infection-lower-women (accessed 4 September 2013).

\section{Richards 2005}

Richards D, Toop L, Chambers S, Fletcher L. Response to antibiotics of women with symptoms of urinary tract infection but negative dipstick urine test results: double blind randomised controlled trial. BMJ 2005;331(7509): 143. [MEDLINE: 15972728]

\section{Spooner 1984}

Spooner JB. Alkalinisation in the management of cystitis. Journal of International Medical Research 1984;12(1):30-4. [MEDLINE: 6692966]

\section{Zhanel 1991}

Zhanel GG, Karlowsky JA, Davidson RJ, Hoban DJ. Influence of human urine on the in vitro activity and postantibiotic effect of ciprofloxacin against Escherichia coli. Chemotherapy 1991;37(3):218-23. [MEDLINE: 1889309]

* Indicates the major publication for the study 
A P P E N DICES

\section{Appendix I. Electronic search strategies}

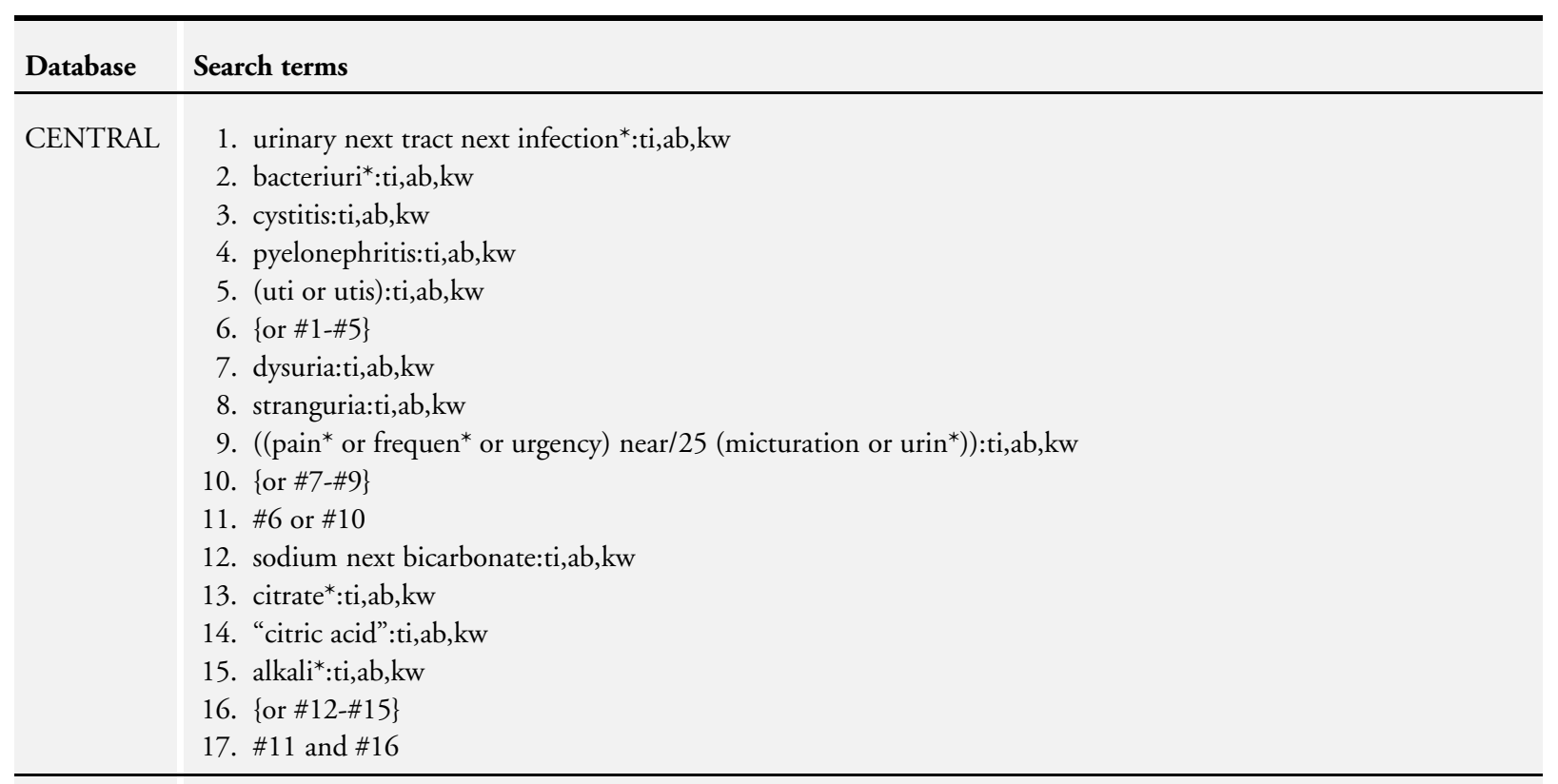

MEDLINE 1. urinary tract infections

2. bacteriuria/

3. cystitis/

4. Pyelonephritis/

5. urinary tract infection*.tw.

6. (uti or utis).tw.

7. bacteriuri*.tw.

8. cystitis.tw.

9. pyelonephritis.tw.

10. or/1-9

11. Dysuria/

12. stranguria.tw.

13. dysuria.tw.

14. ((pain* or frequen* or urgency) adj25 (micturation or urin*)).tw.

15. or/11-14

16. or $/ 10,15$

17. Sodium Bicarbonate/

18. exp Citrates/

19. sodium bicarbonate.tw, nm.

20. citrate.tw,nm.

21. alkali*.tw.

22. or $/ 17-21$

23. and $/ 16,22$ 


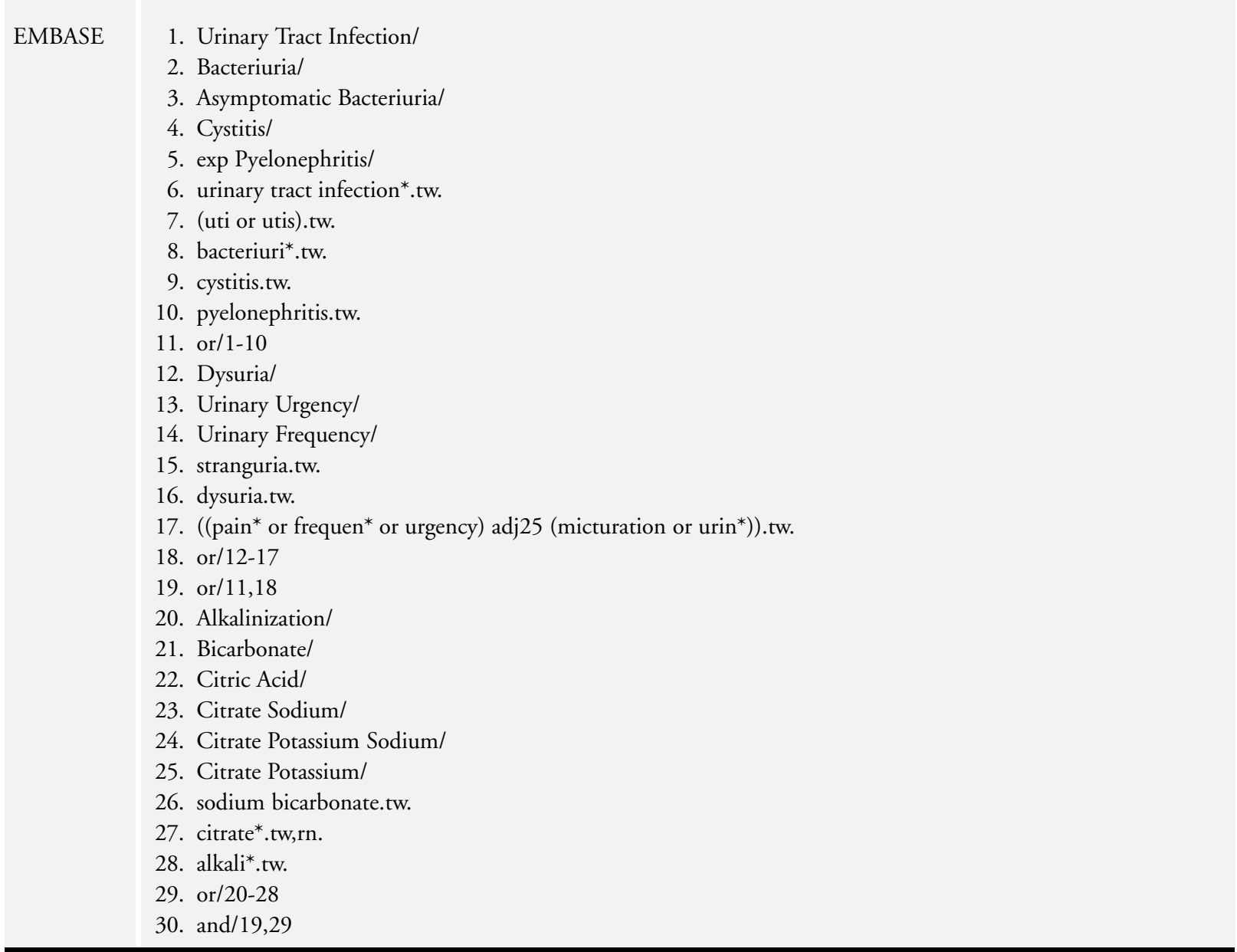

Appendix 2. Risk of bias assessment tool

\section{Potential source of bias}

\section{Random sequence generation}

Selection bias (biased allocation to interventions) due to inadequate generation of a randomised sequence

\section{Assessment criteria}

Low risk of bias: Random number table; computer random number generator; coin tossing; shuffling cards or envelopes; throwing dice; drawing of lots; minimization (minimization may be implemented without a random element, and this is considered to be equivalent to being random)

High risk of bias: Sequence generated by odd or even date of birth; date (or day) of admission; sequence generated by hospital or clinic record number; allocation by judgement of the clinician; by preference of the participant; based on the results of a laboratory 
test or a series of tests; by availability of the intervention

Unclear: Insufficient information about the sequence generation process to permit judgement

\section{Allocation concealment}

Selection bias (biased allocation to interventions) due to inadequate concealment of allocations prior to assignment
Low risk of bias: Randomisation method described that would not allow investigator/participant to know or influence intervention group before eligible participant entered in the study (e.g. central allocation, including telephone, web-based, and pharmacy-controlled, randomisation; sequentially numbered drug containers of identical appearance; sequentially numbered, opaque, sealed envelopes)

High risk of bias: Using an open random allocation schedule (e.g. a list of random numbers); assignment envelopes were used without appropriate safeguards (e.g. if envelopes were unsealed or nonopaque or not sequentially numbered); alternation or rotation; date of birth; case record number; any other explicitly unconcealed procedure

Unclear: Randomisation stated but no information on method used is available

Low risk of bias: No blinding or incomplete blinding, but the review authors judge that the outcome is not likely to be influenced by lack of blinding; blinding of participants and key study personnel ensured, and unlikely that the blinding could have been broken

High risk of bias: No blinding or incomplete blinding, and the outcome is likely to be influenced by lack of blinding; blinding of key study participants and personnel attempted, but likely that the blinding could have been broken, and the outcome is likely to be influenced by lack of blinding

Unclear: Insufficient information to permit judgement

Low risk of bias: No blinding of outcome assessment, but the review authors judge that the outcome measurement is not likely to be influenced by lack of blinding; blinding of outcome assessment ensured, and unlikely that the blinding could have been broken

High risk of bias: No blinding of outcome assessment, and the outcome measurement is likely to be influenced by lack of blinding; blinding of outcome assessment, but likely that the blinding could have been broken, and the outcome measurement is likely to be influenced by lack of blinding

Unclear: Insufficient information to permit judgement 


\section{Incomplete outcome data}

Attrition bias due to amount, nature or handling of incomplete outcome data
Low risk of bias: No missing outcome data; reasons for missing outcome data unlikely to be related to true outcome (for survival data, censoring unlikely to be introducing bias); missing outcome data balanced in numbers across intervention groups, with similar reasons for missing data across groups; for dichotomous outcome data, the proportion of missing outcomes compared with observed event risk not enough to have a clinically relevant impact on the intervention effect estimate; for continuous outcome data, plausible effect size (difference in means or standardized difference in means) among missing outcomes not enough to have a clinically relevant impact on observed effect size; missing data have been imputed using appropriate methods

High risk of bias: Reason for missing outcome data likely to be related to true outcome, with either imbalance in numbers or reasons for missing data across intervention groups; for dichotomous outcome data, the proportion of missing outcomes compared with observed event risk enough to induce clinically relevant bias in intervention effect estimate; for continuous outcome data, plausible effect size (difference in means or standardized difference in means) among missing outcomes enough to induce clinically relevant bias in observed effect size; 'as-treated' analysis done with substantial departure of the intervention received from that assigned at randomisation; potentially inappropriate application of simple imputation

Unclear: Insufficient information to permit judgement

\section{Selective reporting}

Reporting bias due to selective outcome reporting
Low risk of bias: The study protocol is available and all of the study's pre-specified (primary and secondary) outcomes that are of interest in the review have been reported in the pre-specified way; the study protocol is not available but it is clear that the published reports include all expected outcomes, including those that were pre-specified (convincing text of this nature may be uncommon)

High risk of bias: Not all of the study's pre-specified primary outcomes have been reported; one or more primary outcomes is reported using measurements, analysis methods or subsets of the data (e.g. subscales) that were not pre-specified; one or more reported primary outcomes were not pre-specified (unless clear justification for their reporting is provided, such as an unexpected adverse effect); one or more outcomes of interest in the review are reported incompletely so that they cannot be entered in a metaanalysis; the study report fails to include results for a key outcome that would be expected to have been reported for such a study

Unclear: Insufficient information to permit judgement 
Other bias

Bias due to problems not covered elsewhere in the table
Low risk of bias: The study appears to be free of other sources of bias.

High risk of bias: Had a potential source of bias related to the specific study design used; stopped early due to some data-dependent process (including a formal-stopping rule); had extreme baseline imbalance; has been claimed to have been fraudulent; had some other problem

Unclear: Insufficient information to assess whether an important risk of bias exists; insufficient rationale or evidence that an identified problem will introduce bias

\section{CONTRIBUTIONSOFAUTHORS}

1. Draft the protocol: DOK, SD, NG, FP, CDM

2. Study selection: DOK, SD, NG, FP, CDM

3. Extract data from studies: DOK, CMD, TH

4. Enter data into RevMan: DOK

5. Carry out the analysis: DOK, CDM, TH, SD, NG, FP

6. Interpret the analysis: DOK, CDM, TH, SD, NG, FP

7. Draft the final review: DOK

8. Disagreement resolution: $\mathrm{DOK}, \mathrm{CDM}, \mathrm{TH}$

9. Update the review: DOK, CDM, TH, SD, NG, FP

\section{DECLARATIONSOF INTEREST}

None known. 